\title{
Fremanezumab in the Prevention of High-Fr
Episodic and Chronic Migraine: A 12-Week, Multicenter, Real-Life, Cohort Study (The FRIEND Study).
}

\section{Piero Barbanti ( $\nabla$ piero.barbanti@sanraffaele.it)}

IRCCS San Raffaele Pisana: Istituto di Ricovero e Cura a Carattere Scientifico San Raffaele Pisana https://orcid.org/0000-0002-5670-3755

\section{Gabriella Egeo}

IRCCS San Raffaele Pisana: Istituto di Ricovero e Cura a Carattere Scientifico San Raffaele Pisana

Cinzia Aurilia

IRCCS San Raffaele Pisana: Istituto di Ricovero e Cura a Carattere Scientifico San Raffaele Pisana

Florindo d'Onofrio

Presidio Ospedaliero San Giuseppe Moscati

\section{Maria Albanese}

Azienda Ospedaliera Universitaria Policlinico Tor Vergata: Fondazione PTV Policlinico Tor Vergata

\section{Ilaria Cetta}

San Raffaele Hospital: IRCCS Ospedale San Raffaele

\section{Paola Di Fiore}

ASST Santi Paolo e Carlo: Azienda Socio Sanitaria Territoriale Santi Paolo e Carlo

\section{Maurizio Zucco}

San Camillo Forlanini Hospital: Azienda Ospedaliera San Camillo Forlanini

\section{Massimo Filippi}

San Raffaele Hospital: IRCCS Ospedale San Raffaele

\section{Francesco Bono}

Mater Domini University Hospital: Azienda Ospedaliero Universitaria Materdomini

\section{Claudia Altamura}

Policlinico Universitario Campus Bio-Medico

\section{Stefania Proietti}

IRCCS San Raffaele Pisana: Istituto di Ricovero e Cura a Carattere Scientifico San Raffaele Pisana

\section{Stefano Bonassi}

IRCCS San Raffaele Pisana: Istituto di Ricovero e Cura a Carattere Scientifico San Raffaele Pisana

\section{Fabrizio Vernieri}

Policlinico Universitario Campus Bio-Medico 


\section{Research Article}

Keywords: fremanezumab, migraine treatment, CGRP monoclonal antibody, real-world, predictor.

Posted Date: December 28th, 2021

DOI: https://doi.org/10.21203/rs.3.rs-1175049/v1

License: (c) (i) This work is licensed under a Creative Commons Attribution 4.0 International License. Read Full License

Version of Record: A version of this preprint was published at The Journal of Headache and Pain on April 9th, 2022. See the published version at https://doi.org/10.1186/s10194-022-01396-x. 


\section{Abstract \\ Background}

Fremanezumab has demonstrated to be effective, safe, and tolerated in the prevention of episodic or chronic migraine (CM) in randomized, placebo-controlled trials (RCTs). Real-life studies are needed to explore drug effects in unselected patients in routine circumstances and to provide higher generalizability results. This study explores the effectiveness, safety, and tolerability of fremanezumab in a real-life population of individuals affected by high-frequency episodic (HFEM: 8-14 days/month) or CM.

\section{Methods}

This is a 12-week multicenter, prospective, cohort, real-life study. We considered all consecutive patients affected by HFEM or CM visited at 9 Italian headache centers from 28/07/2020 to 11/11/2020. Eligible patients were given subcutaneous fremanezumab at the doses of $225 \mathrm{mg}$ monthly or $675 \mathrm{mg}$ quarterly, according to their preference. Primary study endpoints were the change in monthly migraine days (MMDs) in HFEM and monthly headache days (MHDs) in CM patients at weeks 9-12 compared to baseline. Secondary endpoints encompassed variation in monthly analgesic intake (MAl), Numerical Rating Scale (NRS), HIT-6 and MIDAS scores, and $\geq 50 \%, \geq 75 \%$ and $100 \%$ responder rates at the same time intervals.

\section{Results}

67 migraine patients had received $\geq 1$ subcutaneous fremanezumab dose and were considered for safety analysis, while 53 patients completed 12 weeks of treatment and were included also in the effectiveness analysis. Fremanezumab was effective in both HFEM and CM, inducing at week 12 a significant reduction in MMDs (-4.6, $p<0.05)$, MHDs (-9.4, $p<0.001)$, MAI $(-5.7, p<0.05 ;-11.1, p<0.001)$, NRS (-3.1, $p<0.001 ;-2.5, p<0.001)$, and MIDAS scores $(-58.3, p<0.05 ;-43.7 ; p<0.001)$. HIT-6 was significantly reduced only in HFEM patients $(-18.1, \mathrm{p}<0.001)$. Remission from CM to episodic migraine and from MO to no-MO occurred in $75 \%$ and $67.7 \%$ of the patients. The $\geq 50 \%, \geq 75 \%$ and $100 \%$ responder rates at week 12 were $76.5 \%, 29.4 \%$ and $9.9 \%$ in HFEM and $58.3 \%, 25 \%$ and $0 \%$ in CM. Younger age emerged as a positive response predictor $(\mathrm{OR}=0.91 ; 95 \% \mathrm{Cl} 0.85-0.98, \mathrm{p}=0.013)$. Treatment-emergent adverse events were uncommon (5.7\%) and mild. No patient discontinued fremanezumab for any reason.

\section{Conclusions}

Fremanezumab seems more effective in real-life than in RCTs. Younger age emerges as a potential response predictor. 


\section{Introduction}

Migraine is a complex neurologic disorder characterized by recurrent disabling headache episodes associated with autonomic symptoms [1]. The conventional oral preventive migraine therapies are nonselective, non-specific, poorly tolerated, and burdened by a high discontinuation rate [2-4]. Monoclonal antibodies (mAbs) targeting the calcitonin gene-related peptide (CGRP) - the first specific migraine prophylactic agents - are changing the scene of migraine prevention, coupling promising efficacy to an excellent tolerability profile [5].

Fremanezumab is a humanized mAbs targeting both the $a$ and $\beta$ CGRP isoforms, indicated for the prevention of episodic or chronic migraine in adults. Its peculiarity is the flexible dose regimen which allows to personalize the treatment choosing between the dose of $225 \mathrm{mg}$ on monthly basis or $675 \mathrm{mg}$ quarterly [6]. Fremanezumab has been extensively investigated in randomized, placebo-controlled trials (RCTs) in patients affected by episodic migraine (EM) (HALO-EM study), chronic migraine (CM) (HALOCM study) and EM or CM with 2 to 4 prior therapeutic failures (FOCUS study), documenting a significant superiority over placebo in reducing migraine frequency, analgesic use and disability, and a good efficacy/tolerability profile also in long-term treatment trials [7-10].

While pharmacological RCTs test drugs under ideal conditions, real-life studies explore their effectiveness, safety, and tolerability in unselected patients in routine circumstances, detecting rare or late-onset adverse events, assessing adherence and patterns of use, providing higher generalizability results and testing new hypotheses [11]. Preliminary results from non-peer reviewed publications on retrospective, real-world fremanezumab studies in patients with EM or CM documented a reduction ranging from $68.7 \%$ to $77 \%$ in monthly migraine days (MMDs) and from $65.9 \%$ to $74.8 \%$ in monthly headache days (MHDs), lower acute medication use and emergency department and outpatient physician costs [12].

The present paper is aimed at evaluating the effectiveness, safety, and tolerability of fremanezumab in a prospective real-life, multicenter Italian study in patients affected by high-frequency episodic (HFEM: $\geq 8$ MMDs) or CM.

\section{Methods}

This is a 12-week multicenter, prospective, cohort, real-life study ongoing at 9 headache centers distributed across 4 Italian regions (Lombardy, Latium, Campania, and Calabria) from July $28^{\text {th }}, 2020$, with the latest data analysis performed on November $11^{\text {th }}, 2020$. We considered all consecutive patients affected by HFEM or CM - according to the criteria of the International Classification of Headache Disorders, 3rd edition [13] - with indication to fremanezumab preventive treatment according to the reimbursement rules of the Italian Medicine Agency (AIFA) [14]. None of them was previously treated with any antiCGRP mAbs.

After signing the informed consent, all patients underwent a careful physical and neurological examination and were interviewed using a shared semi-structured questionnaire by specifically trained, 
board-certified neurologists who gathered information on socio-demographic characteristic, migraine features, past and current migraine treatments, comorbidities, and concomitant medications [15].

Patients were given subcutaneous fremanezumab at the doses of $225 \mathrm{mg}$ monthly or $675 \mathrm{mg}$ quarterly, according to their preference. During the 28-day run-in baseline period and the entire study duration, patients were asked to fill-out a paper-pencil diary recording MMDs for HFEM, MHDs for CM, monthly analgesic intake, and rating pain intensity of the monthly most painful attack (0-10, Numerical Rating Scale, NRS). Pain disability was measured monthly using the Headache Impact Test (HIT-6) and quarterly with the Migraine Disability Assessment Scale (MIDAS).

The primary study endpoints were the change in MMDs for HFEM and MHDs for CM at weeks 9-12 compared to baseline. Secondary endpoints encompassed variation in monthly analgesic intake, NRS, HIT- 6 and MIDAS scores and $\geq 50 \%, \geq 75 \%$ and $100 \%$ response rates at the same time intervals. All adverse events (AEs) were evaluated. The study, not preregistered, was approved by the IRCCS San Raffaele Roma Institutional Review Board (RP 19/26) as coordinating center and mutually recognized by the other local Institutional Review Boards.

\section{Statistical methods}

Categorical variables in demographic and clinical data were reported as percentages, and group differences were assessed using the $\chi^{2}$ test or the Fisher exact test (2-tailed). Continuous data was summarized as mean and standard deviation (SD) for descriptive purposes. The comparison of post treatment values with baseline was done with the paired $t$-test or non-parametric Wilcoxon test for paired data, while the comparison of groups of patients with episodic or chronic migraine was done with the independent Student's $t$-test or by the Mann Whitney $U$ test if the distribution of the data was non normal. The Shapiro-Wilk test was applied to test the departure from normality of data distribution. A multivariate logistic regression models was fitted to identify factors associated with the response. Potential confounders and variables which were statistically significant in the univariate analysis were included in the models. Risks were expressed as odds ratio (OR) along with its $95 \%$ confidence interval $(95 \% \mathrm{Cl})$. Results were considered statistically significant when $p<0.05$. SPSS (IBM SPSS Statistics for Windows, version 27.0) and GraphPad Prism (GraphPad Software, Inc; v8.00.) statistical software was used for statistical analysis.

\section{Results}

Sixty-seven migraine patients (HFEM, $n=21 ; C M, n=46 ; F / M ~ 53 / 14$; mean age 48.4 years) at November $11^{\text {th }} 2020$ had received at least one subcutaneous fremanezumab dose $(225 \mathrm{mg}$ monthly, $\mathrm{n}=58 ; 625 \mathrm{mg}$ quarterly, $n=9$ ) and were considered for safety analysis, while 53 patients completed 12 weeks of treatment and were included in the effectiveness analysis (figure 1). Table 1 summarizes their demographic and clinical features. Most patients were females $(41 / 53,77.3 \%)$, affected by CM $(36 / 53$, $67.9 \%$ ), with concomitant medication overuse (MO: $31 / 36,86.1 \%)$ and showed on average 4.5 prior 
preventative failures. The only variable which showed significant difference between the two groups was the monthly analgesic intake, which was nearly double in CM as compared to HFEM $(22.4 \pm 18.5$ vs $11.9 \pm 7.1 ; p<0.029)$. Only 9 patients $(17 \%)$ were treated with a quarterly fremanezumab dosing regimen.

Fremanezumab was effective in both HFEM and CM patients (figure 2, supplementary table 1 ). At weeks 4, 8 and 12, fremanezumab induced in HFEM patients a significant reduction in MMDs $(-5.6 \pm 2.9, p<0.001$; $-6.1 \pm 3.6, p<0.001 ;-4.6 \pm 6.5, p<0.05)$, monthly analgesic use $(-7.2 \pm 6.7, p<0.001 ;-7.5 \pm 5.8, p<0.001 ;-5.7 \pm 6.6$, $p<0.05)$, NRS score $(-2.5 \pm 2.5, p<0.05 ;-2.9 \pm 1.9, p<0.001 ;-3.1 \pm 2.5, p<0.001)$ and HIT-6 score $(-4.1 \pm 10.2, n s$; $-12.3 \pm 5.5, p<0.001 ;-18.1 \pm 13.2, p<0.001)$. At the same time intervals, fremanezumab significantly $(p<0.001$ for all) reduced MHDs $(-8.2 \pm 6.1 ;-8.3 \pm 6.8 ;-9.4 \pm 6.9)$, monthly analgesic use $(-9.6 \pm 13.1 ;-8.2 \pm 9.2$; $-11.1 \pm 14.2)$ and NRS score $(-1.7 \pm 1.8 ;-1.7 \pm 1.7 ;-2.5 \pm 2.7)$ in patients with CM. HIT-6 scores did not significantly vary in CM $(-4.5 \pm 21.3 ;-1.2 \pm 20.1 ; 0.3 \pm 23.3)$. MIDAS score at weeks $9-12$ was significantly reduced in HFEM $(-58.3 \pm 57.7 ; p<0.05)$ and CM patients $(-43.7 \pm 63.4 ; p<0.001)$ (figure 2 , supplementary table 1).

Sixty-one-point-one percent (22/36) of CM patients remitted to episodic migraine at week $4,72.2 \%$ $(26 / 36)$ at week 8 , and $75 \%(27 / 36)$ at week 12 , while $61.2 \%(19 / 31)$ of the patients with MO remitted to no-MO at week $4,64.5 \%(20 / 31)$ at week 8 , and $67.7 \%(21 / 31)$ at week 12. Remission from CM to episodic migraine was sustained across week 8 in 100\% (22/22), and week 12 in $95.5 \%$ (21/22) of the patients, while remission from MO to no-MO was sustained in $94.7 \%(18 / 19)$, and $94.4 \%(17 / 18)$ of the cases, respectively (table 2 ).

The $\geq 50 \%, \geq 75 \%$ and $100 \%$ responder rates at week 12 were $76.5 \%, 29.4 \%$ and $9.9 \%$ in HFEM and $58.3 \%$, $25 \%$ and $0 \%$ in CM patients (figure 3). Safety and tolerability data were provided by 60 out of the 67 patients treated with $\geq 1$ fremanezumab dose because at the time of data analysis 7 patients had not yet performed the first follow-up visit, scheduled 4 weeks after the first dose administration. Treatmentemergent adverse events, rated as mild and transient, were reported by 1 patient at week $4(1.7 \%), 2$ at week 8 (3.4\%) and $3(5.7 \%)$ at week 12 (table 3 ). The most common was injection site erythema. No patient discontinued fremanezumab treatment for any reason.

The univariate analysis of independent determinant of $\geq 50 \%$ response documented that responders were significantly younger ( $44.3 \pm 11.3$ vs $53.8 \pm 9.4$ years; $p=0.03$ ), had lower monthly analgesic intake ( $15.3 \pm 15.0$ vs $25.7 \pm 17.3 ; p=0.026)$, shorter medication overuse duration ( $13.8 \pm 12.1$ vs $40.4 \pm 49.5$ months; $p<0.041)$, and more frequent use of the monthly dosing regimen ( $75 \%$ vs $11 \% ; p=0.001)$ (table 4$)$.

When stratifying patients according to migraine frequency, younger age and shorter medication overuse emerged as positive predictors only in $\mathrm{CM}$ ( $\mathrm{p}<0.001$ and 0.041 , respectively), whereas lower monthly analgesic intake was found only in HFEM patients $(p=0.046)$. Monthly dosing regimen was associated to higher probability of fremanezumab responsiveness in both HFCM $(p=0.022)$ and CM patients $(p=0.008)$, although the small number of responders among those treated quarterly makes these statistics highly unstable. Lastly, CM patients with lower HIT-6 score were more likely to be fremanezumab responders $(p=0.038)$. To consider the role of confounders, a multivariate logistic regression analysis model was 
fitted to data. The only variable which survived the backward process was age $(\mathrm{OR}=0.91 ; 95 \% \mathrm{Cl} 0.85$ $0.98, p=0.013)$, confirming the results of univariate analysis, which showed a better response rate in younger patients. The effect of fremanezumab dosing regimen could not be properly estimated because of the small number of subjects treated quarterly.

\section{Discussion}

Real-life studies are needed to translate the evidence concerning the efficacy of antiCGRP mAbs into effectiveness, to confirm their safety and tolerability, and to identify response predictors in a multifaceted clinical setting. This approach is particularly valuable to study patients with various comorbidities and inadequate response to diverse classes of prior preventive treatments.

The present prospective, multicenter, real-life Italian study documents that fremanezumab is effective, safe, and well tolerated in the prevention of subjects affected by difficult-to-treat HFEM or CM with multiple therapeutic failures. These results demonstrate an early and progressive improvement in migraine frequency, analgesic use, pain intensity, and MIDAS score in these patients. Of note, the treatment induced the remission from $\mathrm{CM}$ to episodic migraine and from $\mathrm{MO}$ to no-MO in over two-thirds of the patients, the improvement being persistent over 12 weeks in almost all cases. Adverse events were extremely rare, and no patient discontinued the treatment for any reason.

In published RCTs focusing on patients with $<2$ prior therapeutic failures, fremanezumab demonstrated to be significantly superior to placebo in reducing MMDs (HALO-EM: $-3.7 /-3.4$ vs $-2.2 ; p<0.001$ ) and MHDs (HALO-CM: $-4.6 /-4.3 ; p<0.001$ ) as well as in $\geq 50 \%$ responder rate (HALO-EM: $47.7 \% / 44.4 \%$ vs $27.9 \%$, p $<0.0001$; HALO-CM: $40.8 \% / 37.6 \%$ vs $18.1 \% ; p<0.0001$ ), revealing also a good safety and tolerability profile [7-8]. Interestingly, fremanezumab proved an even better efficacy/tolerability ratio in patients who had not responded to 2-4 preventive medication clusters (FOCUS study), documenting a higher therapeutic gain over placebo in terms of MMDs/MHDs change $(-4.1 /-3.7$ vs $-0.6 ; p<0.0001)$ and $\geq 50 \%$ responder rate ( $34 \%$ vs $9 \%, \mathrm{p}<0.0001$ ) when compared to the HALO-EM and HALO-CM trials, coupled to a lower incidence of adverse events (45\%-55\% vs $66.2 \%-66.3 \%$ and $70 \%-71 \%$, respectively) [9].

Patients considered in the FRIEND study were harder to treat than those included in the FOCUS trial because almost all of them had failed $\geq 3$ preventive treatments (93\% vs $53 \%$ ), had higher migraine frequency (18.9 days/month vs 14.3 days/month), were more frequently affected by CM $(67.9 \%$ vs $60.1 \%$ ) and MO ( 86.1 vs $52 \%$ ), and showed higher disability (MIDAS score 89.4 vs 61.8 ). Further, among episodic migraine patients, we considered only HFEM, i.e., those with $\geq 8$ MMDs.

A direct comparison of real-life data with the results of RCTs is not scientifically accurate, especially when the number of patients studied is heterogeneous. This notwithstanding, it's worth mentioning that at weeks 9-12 the reduction in MMDs/MHDs and the proportion of $\geq 50 \%$ responders in our patients were greater than those reported in the FOCUS study $(-7.9$ vs $-3.7 /-4.1 ; 64.2 \%$ vs $34 \%$, respectively), suggesting that the effectiveness of fremanezumab could be better than its efficacy - an aspect already reported for erenumab and galcanezumab [15-19]. 
Why antiCGRP mAbs seem to work better in migraine patients with more complex clinical picture (as the real-life ones) compared to those enrolled in the RCTs, is matter of speculation. This could depend - at least in part - on their higher baseline MMDs/MHDs. As a matter of fact, migraine frequency positively correlates with CGRP plasma levels, which are higher in CM than in episodic migraine patients and healthy controls [20]. Notably, even among real-life patients, we documented a better antiCGRP mAbs responsiveness in those with higher baseline migraine frequency (OR: 1.12; 95\% Cl: 1.05-1.20, $\mathrm{p}<0.001$ ) [16]. Another possible reason is the high proportion of patients with allodynia in real-life $(59.2 \%$ in our study). Allodynia is seen as a CGRP-related symptom and prevails in patients with long-lasting attacks, long disease history, severe pain intensity and disability, MO, and psychiatric comorbidities, features typically characterizing the real-world setting [21-23]. For the above reasons, it can be argued that real-life patients could be characterized by a greater CGRP pathogenetic involvement, thus being more sensitive to CGRP-targeting treatments.

In the FRIEND study, fremanezumab significantly improved MIDAS score in all the patients, but reduced HIT-6 only in those with HFEM. We have no reliable explanation for this controversial finding, probably biased by the small number of patients studied. MIDAS and HIT-6 are complimentary in measuring migraine-related disability but also show some substantial differences. MIDAS is basically a function of migraine frequency and relies on concrete variables (i.e., days of work or school missed), whilst HIT-6 depends more on headache severity and explores patients' impressions on how migraine is affecting them [24].

Consequently, we cannot exclude that the above discrepancy is also somehow related to the different benefits produced by fremanezumab on migraine frequency and intensity in our patients' groups. Indeed, at week 12, the reduction in MMDs/MHDs was comparable in HFEM $(-47 \%)$ and CM $(-43.8 \%)$, whereas the decrease in intensity (NRS) was more evident in episodic (-36.5\%) than in chronic migraine patients $(-29 \%)$.

Age came up as a potential negative response predictor in our study $(\mathrm{OR}=0.91 ; 95 \% \mathrm{Cl} 0.85-0.98$, $p<0.013$ ). The odd of being fremanezumab responder would decrease by $9 \%$ for each year of age, suggesting that its effectiveness could be higher in younger patients. This assumption, however, must be considered with caution, because the population studied is small and mostly represented by CM patients. Similarly, the apparent clinical advantage of the monthly dosing regimen over the quarterly one cannot be established because most patients (83\%) had preferred a monthly $225 \mathrm{mg}$ fremanezumab dose.

The results of the present study should be interpreted with caution due to some limitations. The patient sample is small, due to limited pre-reimbursement access to fremanezumab in our Country. In addition, patients reported migraine data using paper-pencil diaries, a less reliable tool compared to modern electronic diaries. Strengths are the multicenter prospective design, the involvement of 9 headache centers representative of northern, central, and southern Italy and a careful clinical characterization of the patients through a shared detailed semi-structured questionnaire. 
In conclusion, our multicenter, prospective study indicates that fremanezumab is effective and extremely tolerated also in real-life subjects affected by HFEM or CM with multiple therapeutic failures. Our results deserve confirmation in larger real-life studies.

\section{Abbreviations}

CM: Chronic migraine; RCTs: Placebo-controlled trials; HFEM: High-frequency episodic; MMDs: monthly migraine days; MAl: monthly analgesic intake; NRS: Numerical Rating Scale, HIT-6: Headache Impact Test; MIDAS: Migraine Disability Assessment Scale; mAbs: Monoclonal antibodies; CGRP: Calcitonin gene-related peptide; EM: Episodic migraine, AEs: Adverse events; SD: Standard deviation.

\section{Declarations}

\section{Acknowledgements}

FRIEND Study Group Collaborators: Fabio Bombardieri ${ }^{9}$, Nicoletta Brunelli ${ }^{10}$, Bruno Colombo ${ }^{5}$, Carmelina Maria Costa ${ }^{10}$, Lara Di Clemente ${ }^{7}$, Luigi d'Onofrio ${ }^{14}$, Luisa Fofi ${ }^{1}$, Giulia Fiorentini ${ }^{2}$, Fabio Frediani ${ }^{6}$, Domenica Le Pera ${ }^{1,15}$, Giuseppe Magro ${ }^{9}$, Alessandro Mechelli ${ }^{9}$, Nicola Biagio Mercuri ${ }^{4}$, Roberta Messina ${ }^{5}$, Daniele Spitaleri ${ }^{3}$, Carlo Tomino ${ }^{15}$, Giovanna Viticchi ${ }^{16}$.

\section{Authors' contributions}

PB and FV designed the study, GE and CA drafted the manuscript, SB and SP carried out data analysis, GE, CA, FdO, MA, IC, PDF, MZ FB, CA and FRIEND-Study Group performed data collection, MF, PB and FV revised the manuscript. The author(s) read and approved the final manuscript.

\section{Funding.}

This work was partially supported by the Italian Ministry of Health (Institutional Funding Ricerca Corrente) IRCCS San Raffaele Roma.

\section{Availability of data and materials}

Anonymized data will be shared by request from any qualified investigator

Ethics approval and consent to participate All patients provided written informed consent. The study was approved by IRCCS San Raffaele Rome Ethical Committee n RP 19/26, mutually recognized by the other local ethical committees.

\section{Conflict of Interest:}

Piero Barbanti received travel grants, honoraria for advisory boards, speaker panels or clinical investigation studies from Alder, Allergan, Angelini, Assosalute, Bayer, ElectroCore, Eli-Lilly, GSK, 
Lundbeck, Lusofarmaco, 1MED, MSD, New Penta, Noema Pharma, Novartis, Stx-Med, Teva, Visufarma, Zambon.

Cinzia Aurilia received travel grants from FB-Health, Lusofarmaco, Almirall, Eli-Lilly Novartis and Teva; Gabriella Egeo received travel grants and honoraria from Eli-Lilly, Novartis, New Penta and Ecupharma; Florindo d'Onofrio received travel grant, honoraria as a speaker or for partecipating in advisory boards from Novartis, Teva, Neopharmed Gentili, Qbgroup srl, K link srl and Eli-Lilly.

Maria Albanese received travel grants and honoraria from Novartis, Teva, Eli-Lilly and Lundbeck.

Ilaria Cetta have no disclosures to declare

Paola Di Fiore received honoraria as a speaker or for participating in advisory boards from Novartis and Teva.

Maurizio Zucco received travel grants and honoraria from Novartis

Massimo Filippi is Editor-in-Chief of the Journal of Neurology; received compensation for consulting services and/or speaking activities from Bayer, Biogen Idec, Merck-Serono, Novartis, Roche, Sanofi Genzyme, Takeda, and Teva Pharmaceutical Industries; and receives research support from Biogen Idec, Merck-Serono, Novartis, Roche, Teva Pharmaceutical Industries, Italian Ministry of Health, Fondazione Italiana Sclerosi Multipla, and ARiSLA (Fondazione Italiana di Ricerca per la SLA).

Francesco Bono received honoraria as a speaker or for participating in advisory boards from Teva, Novartis and Ipsen.

Claudia Altamura received travel grants or honoraria for speaker panels from Eli-Lilly, Novartis, Teva, Lusofarmaco, Almirall, Laborest.

S. Proietti have no disclosures to declare.

S. Bonassi have no disclosures to declare.

Fabrizio Vernieri received travel grants, honoraria for advisory boards, speaker panels or clinical investigation studies from Allergan, Eli-Lilly, Novartis, Teva, Amgen, Angelini and Lundbeck.

\section{References}

1. Ashina M. Migraine. N Engl J Med. 2020 Nov 5;383(19):1866-1876.

2. Parikh SK, Silberstein SD. Preventive Treatment for Episodic Migraine. Neurol Clin. 2019; Nov;37(4):753-770. doi: 10.1016/j.ncl.2019.07.004. Epub 2019 Aug 22. 
3. Lipton RB, Silberstein SD. Episodic and chronic migraine headache: breaking down barriers to optimal treatment and prevention. Headache. 2015 Mar;55 Suppl 2:103-22.

4. Agostoni EC, et al. Current and emerging evidence-based treatment options in chronic migraine: a narrative review. J Headache Pain. 2019 Aug 30;20(1):92. doi: 10.1186/s10194-019-1038-4

5. Alasad YW, Asha MZ. Monoclonal antibodies as a preventive therapy for migraine: A metaanalysis. Clin Neurol Neurosurg. 2020 Aug;195:105900. doi: 10.1016/j.clineuro.2020.105900. Epub 2020 May

6. Hoy SM. Fremanezumab: First Global Approval. Drugs. 2018 Nov;78(17):1829-1834. doi: 10.1007/s40265-018-1004-5.

7. Dodick DW, Silberstein SD, Bigal ME, et al. Effect of fremanezumab compared with placebo for prevention of episodic migraine: a randomized clinical trial. JAMA. 2018;319(19):1999-2008.

8. Silberstein SD, Dodick DW, Bigal ME, et al. Fremanezumab for the preventive treatment of chronic migraine. N Engl J Med. 2017;377(22):2113-2122.

9. Ferrari MD, Diener HC, Ning X, Galic M, Cohen JM, Yang R, Mueller M, Ahn AH, Schwartz YC, Grozinski-Wolff M, Janka L, Ashina M. Fremanezumab versus placebo for migraine prevention in patients with documented failure to up to four migraine preventive medication classes (FOCUS): a randomised, double-blind, placebo-controlled, phase 3b trial. Lancet. 2019 Sep 21;394(10203):10301040.

10. Goadsby PJ, Silberstein SD, Yeung PP, Cohen JM, Ning X, Yang R, Dodick DW. Long-term safety, tolerability, and efficacy of fremanezumab in migraine: A randomized study. Neurology. 2020 Nov 3;95(18): e2487-e2499. doi: 10.1212/WNL.0000000000010600. Epub 2020 Sep 10.

11. Saturni S, Bellini F, Braido F, Paggiaro P, Sanduzzi A, Scichilone N, Santus PA, Morandi L, Papi A. Randomized Controlled Trials and real life studies. Approaches and methodologies: a clinical point of view. Pulm Pharmacol Ther. 2014 Apr;27(2):129-38

12. Cohen JM, Thompson S, Patterson-Lomba O, Driessen MT, Seminerio M, Carr K, Mu F. US real-world reductions in migraine and headache days for patients with chronic and episodic migraine initiating fremanezumab. Abstract. Presented at the 2021 American Academy of Neurology Annual Meeting.

13. Headache Classification Committee of the International Headache Society. The International Classification of Headache Disorders, 3rd edition (beta version). Cephalalgia 2013;33:629-808

14. Gazzetta Ufficiale n.182, 21-7-2020

15. Barbanti P, Aurilia C, Egeo G, Fofi L, Cevoli S, Colombo B, Filippi M, Frediani F, Bono F, Grazzi L, Salerno A, Mercuri B, Carnevale A, Altamura C, Vernieri F. Erenumab in the prevention of highfrequency episodic and chronic migraine: Erenumab in Real Life in Italy (EARLY), the first Italian multicenter, prospective real-life study. Headache. 2021 Feb;61(2):363-372.

16. Barbanti P, Aurilia C, Cevoli S, Egeo G, Fofi L, Messina R, Salerno A, Torelli P, Albanese M, Carnevale A, Bono F, D'Amico D, Filippi M, Altamura C, Vernieri F; EARLY Study Group. Long-term (48 weeks) effectiveness, safety, and tolerability of erenumab in the prevention of high-frequency episodic and chronic migraine in a real world: Results of the EARLY 2 study. Headache. 2021 Oct;61(9):1351-1363. 
17. Vernieri F, Altamura C, Brunelli N, Costa CM, Aurilia C, Egeo G, Fofi L, Favoni V, Pierangeli G, Lovati C, Aguggia M, d'Onofrio F, Doretti A, Di Fiore P, Finocchi C, Rao R, Bono F, Ranieri A, Albanese M, Cevoli S, Barbanti P; GARLIT Study Group. Galcanezumab for the prevention of high frequency episodic and chronic migraine in real life in Italy: a multicenter prospective cohort study (the GARLIT study). J Headache Pain. 2021 May 3;22(1):35. doi: 10.1186/s10194-021-01247-1

18. Vernieri F, Altamura C, Brunelli N, Costa CM, Aurilia C, Egeo G, Fofi L, Favoni V, Lovati C, Bertuzzo D, d'Onofrio F, Doretti A, Di Fiore P, Finocchi C, Schiano Di Cola F, Ranieri A, Colombo B, Bono F, Albanese M, Cevoli S, Barbanti P for the GARLIT Study Group. Rapid response to galcanezumab and predictive factors in chronic migraine patients: A 3-month observational, longitudinal, cohort, multicenter, Italian real-life study. European Journal of Neurology, in press.

19. Vernieri F, Brunelli N, Messina R, Costa CM, Colombo B, Torelli P, Quintana S, Cevoli S, Favoni V, d'Onofrio F, Egeo G, Rao R, Filippi M, Barbanti P, Altamura C. Discontinuing monoclonal antibodies targeting CGRP pathway after one-year treatment: an observational longitudinal cohort study. J Headache Pain, in press.

20. Cernuda-Morollon E, Larrosa D, Ramòn C, Vega J, Martínez-Camblor P, Pascual J. Interictal increase of CGRP levels in peripheral blood as a biomarker for chronic migraine. Neurology 2013; 81: 11911196.

21. Durham PL, Vause CV. Calcitonin gene-related peptide (CGRP) receptor antagonists in the treatment of migraine. CNS Drugs. 2010 Jul;24(7):539-48.

22. Schürks M, Diener HC. Migraine, allodynia, and implications for treatment. Eur J Neurol. 2008 Dec;15(12):1279-85

23. Dodick DW, Reed ML, Fanning KM, Munjal S, Alam A, Buse DC, Schwedt TJ, Lipton RB. Predictors of allodynia in persons with migraine: Results from the Migraine in America Symptoms and Treatment (MAST) study. Cephalalgia. 2019 Jun;39(7):873-882

24. Sauro KM, Rose MS, Becker WJ, Christie SN, Giammarco R, Mackie GF, Eloff AG, Gawel MJ. HIT-6 and MIDAS as measures of headache disability in a headache referral population. Headache. 2010 Mar;50(3):383-95.

\section{Tables}

Table 1. Demographic and clinical features of patients with high-frequency episodic migraine (HFEM) or chronic migraine $(\mathrm{CM})$. 


\begin{tabular}{|c|c|c|c|c|}
\hline & All Patients & HFEM & $\mathrm{CM}$ & p-value \\
\hline Patients & 53 & 17 & 36 & \\
\hline Age, $y r s$, mean $\pm S D$ & $47.7 \pm 11.5$ & $47.5 \pm 11.6$ & $47.9 \pm 11.6$ & ns \\
\hline Females, n (\%) & $41(77.3)$ & $12(70.5)$ & $29(80.5)$ & ns \\
\hline BMI, mean $\pm S D$ & $24.0 \pm 3.6$ & $23.6 \pm 2.4$ & $24.2 \pm 4.1$ & ns \\
\hline Age at $\mathrm{CM}$ onset, mean $\pm \mathrm{SD}$ & & - & $34.0 \pm 13.1$ & - \\
\hline Disease duration, $y r s$, mean $\pm S D$ & $29.6 \pm 13.9$ & $29.1 \pm 13.0$ & $29.8 \pm 14.4$ & ns \\
\hline MMDs/MHDs at baseline, mean $\pm S D$ & $17.0 \pm 6.2$ & $10.5 \pm 1.8$ & $20.0 \pm 5.2$ & - \\
\hline NRS score, mean $\pm S D$ & $8.6 \pm 1.1$ & $8.5 \pm 1.2$ & $8.6 \pm 1.1$ & ns \\
\hline Pain location, $\mathrm{n}(\%)$ & & & & ns \\
\hline Unilateral & $21(44.7)$ & $8(50)$ & $13(41.9)$ & \\
\hline Unilateral, bilateral & $23(48.9)$ & $8(50)$ & $15(48.4)$ & \\
\hline Bilateral & $3(6.4)$ & 0 & $3(9.7)$ & \\
\hline Pain quality, n (\%) & & & & ns \\
\hline Pulsating & $28(57.1)$ & $8(53.3)$ & $20(58.8)$ & \\
\hline Pressing/tightening & $12(24.5)$ & $3(20.0)$ & $9(26.5)$ & \\
\hline Other & $9(18.4)$ & $4(26.7)$ & $5(14.7)$ & \\
\hline Unilateral cranial autonomic symptoms, $n(\%)$ & $33(62.2)$ & $12(70.5)$ & $21(58.3)$ & ns \\
\hline Allodynia, $\mathrm{n}(\%)$ & $29(59.2)$ & $10(66.7)$ & $19(55.8)$ & ns \\
\hline Dopaminergic symptoms, $\mathrm{n}(\%)$ & $30(61.2)$ & $10(66.7)$ & $20(58.8)$ & ns \\
\hline Monthly analgesic intake, mean $\pm S D$ & $19.0 \pm 16.5$ & $11.9 \pm 7.1$ & $22.4 \pm 18.5$ & 0.029 \\
\hline MO, n (\%) & & - & $31(86.1)$ & - \\
\hline Duration of MO, $y r s$, mean $\pm S D$ & & - & $24.8 \pm 35.0$ & - \\
\hline Triptan responders, $\mathrm{n}(\%)$ & $34(64.1)$ & $12(70.6)$ & $22(61.1)$ & ns \\
\hline Pts using concomitant prophylaxis, $\mathrm{n}(\%)$ & $29(54.7)$ & $7(41.1)$ & $22(61.1)$ & ns \\
\hline Tricyclics & $10(34.5)$ & $2(28.5)$ & $8(36.4)$ & \\
\hline Anticonvulsants & $7(24.1)$ & $2(28.5)$ & $5(22.7)$ & \\
\hline Calcium-antagonists & $1(3.4)$ & 0 & $1(4.5)$ & \\
\hline Serotoninergic antagonists & $5(17.2)$ & 0 & $5(22.7)$ & \\
\hline
\end{tabular}




\begin{tabular}{|lllll|} 
Beta-blockers & $9(31.0)$ & $2(28.5)$ & $7(31.8)$ & \\
BoNT/A & $2(6.9)$ & 0 & $2(9.1)$ & \\
Other & $6(20.7)$ & $2(28.5)$ & $4(18.2)$ & \\
\hline Prior treatment failures, mean \pm SD & $4.5 \pm 2.3$ & $3.9 \pm 1.5$ & $4.7 \pm 2.5$ & $\mathrm{~ns}$ \\
$1-2$ & $4(8.0)$ & $3(17.7)$ & $1(3.0)$ & \\
$3-4$ & $30(60.0)$ & $10(58.8)$ & $20(60.6)$ & \\
$>4$ & $16(32.0)$ & $4(23.5)$ & $12(36.4)$ & \\
\hline BoNT/A responders ${ }^{a}, \mathrm{n}(\%)$ & $2(11.1)$ & $2(33.3)$ & 0 & 0.098 \\
\hline Pts with $\geq 1$ comorbidity, $\mathrm{n}(\%)$ & $34(64.2)$ & $14(82.4)$ & $20(55.5)$ & 0.072 \\
\hline Pts with psychiatric comorbidities, $\mathrm{n}(\%)$ & $10(19.2)$ & $3(18.8)$ & $7(19.4)$ & $\mathrm{ns}$ \\
\hline HIT-6 score, mean $\pm S D$ & $65.2 \pm 17.2$ & $68.2 \pm 3.4$ & $63.7 \pm 20.8$ & $\mathrm{~ns}$ \\
\hline MIDAS score, mean $\pm S D$ & $89.4 \pm 48.9$ & $78.9 \pm 50.5$ & $94.5 \pm 48.1$ & $\mathrm{~ns}$ \\
\hline Fremanezumab dosing regimen, $\mathrm{n}(\%)$ & & & & $\mathrm{ns}$ \\
\hline Monthly & $44(83.0)$ & $13(76.5)$ & $31(86.1)$ & \\
\hline Quarterly & $9(17.0)$ & $4(23.5)$ & $5(13.9)$ & \\
\hline
\end{tabular}

HFEM: high frequency episodic migraine; $\mathrm{CM}=$ chronic migraine; $\mathrm{BMI}$ : body mass index; MMDs: monthly migraine days; MHDs: monthly headache days; $\mathrm{NRS}=$ numerical rating scale; $\mathrm{MO}=$ medication overuse; BoNT/A=onabotulinum toxin A; HIT-6: Headache Impact Test-6; MIDAS=migraine disability assessment

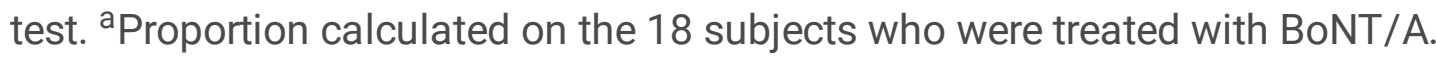

Table 2: Patients remitting from chronic migraine (CM) to episodic migraine (EM) and from medication overuse (MO) to no medication overuse (no-MO) across weeks 4, 8 and 12 following fremanezumab treatment.

\begin{tabular}{|llll|}
\hline & Week 4 & Week 8 & Week 12 \\
\hline CM remission to EM & $22 / 36(61.1 \%)$ & $26 / 36(72.2 \%)$ & $27 / 36(75 \%)$ \\
Sustained remission across weeks 4, 8, 12 & - & $22 / 22(100 \%)$ & $21 / 22(95.5 \%)$ \\
\hline MO remission to no-MO & $19 / 31(61.2 \%)$ & $20 / 31(64.5 \%)$ & $21 / 31(67.7 \%)$ \\
Sustained remission across weeks 4, 8, 12 & - & $18 / 19(94.7 \%)$ & $17 / 19(94.4 \%)$ \\
\hline
\end{tabular}


Table 3. Treatment-emergent adverse event (TEAEs) occurring at weeks 4, 8 and 12.

\begin{tabular}{|llll|}
\hline & Week 4 & Week 8 & Week 12 \\
\hline Patients $n$ & 60 & 58 & 53 \\
\hline Patients with $\geq 1$ TEAE & $1(1.7 \%)$ & $2(3.4 \%)$ & $3(5.7 \%)$ \\
- Injection site erythema & $1(1.7 \%)$ & $1(1.7 \%)$ & $1(1.9 \%)$ \\
- Dizziness & - & $1(1.7 \%)$ & - \\
- Abdominal pain & - & - & $1(1.9 \%)$ \\
- Neck pain and somnolence & - & - & $1(1.0 \%)$ \\
\hline Discontinuation due to TEAEs & - & - & - \\
\hline Discontinuation due to ineffectiveness & - & - & - \\
\hline
\end{tabular}

Table 4. Univariate analysis of independent determinant of $\geq 50 \%$ response 


\begin{tabular}{|c|c|c|c|}
\hline & $<50 \%$ response & $\geq 50 \%$ response & $p$-value \\
\hline Patients, n (\%) & $19(35.8)$ & $34(64.2)$ & - \\
\hline Age, yrs, mean $\pm S D$ & $53.8 \pm 9.4$ & $44.3 \pm 11.3$ & 0.03 \\
\hline Females, n (\%) & $14(34.1)$ & $27(65.9)$ & ns \\
\hline BMI, mean $\pm S D$ & $24.4 \pm 3.9$ & $23.7 \pm 3.5$ & ns \\
\hline Age at $\mathrm{CM}$ onset, mean $\pm \mathrm{SD}$ & $37.2 \pm 15.4$ & $31.9 \pm 11.2$ & ns \\
\hline Disease duration, mean $\pm S D$ & $31.5 \pm 15.0$ & $28.5 \pm 13.3$ & ns \\
\hline MMDs/MHDs at baseline, mean $\pm S D$ & $18.9 \pm 6.3$ & $15.9 \pm 6.0$ & ns \\
\hline NRS score, mean $\pm S D$ & $8.6 \pm 1.3$ & $8.5 \pm 1.0$ & ns \\
\hline Pain location, $\mathrm{n}(\%)$ & & & ns \\
\hline Unilateral & $8(44.4)$ & $13(44.8)$ & \\
\hline Unilateral, bilateral & $8(44.4)$ & $15(51.7)$ & \\
\hline Bilateral & $2(11.2)$ & $1(3.5)$ & \\
\hline Pain quality, n (\%) & & & ns \\
\hline Pulsating & $9(56.3)$ & 19(57.6) & \\
\hline Pressing/tightening & $6(37.5)$ & $6(18.2)$ & \\
\hline Other & $1(6.2)$ & $8(24.2)$ & \\
\hline UAs, n (\%) & $9(56.3)$ & $24(72.7)$ & ns \\
\hline Allodynia, n (\%) & $10(62.5)$ & $19(57.6)$ & ns \\
\hline Dopaminergic symptoms, $\mathrm{n}(\%)$ & $10(62.5)$ & $20(60.6)$ & ns \\
\hline MAI at baseline, mean $\pm S D$ & $25.7 \pm 17.3$ & $15.3 \pm 15.0$ & 0.026 \\
\hline MO, n (\%) & $13(86.7)$ & 18 (85.7) & ns \\
\hline Duration of MO, months; mean $\pm S D$ & $40.4 \pm 49.5$ & $13.8 \pm 12.1$ & 0.041 \\
\hline Triptan responders, n (\%) & $12(63.2)$ & $22(64.7)$ & ns \\
\hline Pts using concomitant prophylaxis, $n$ (\%) & $11(57.9)$ & $18(52.9)$ & ns \\
\hline Tricyclics & $4(36.4)$ & $6(33.3)$ & \\
\hline Anticonvulsants & $3(27.3)$ & $3(16.7)$ & \\
\hline Calcium-antagonists & 0 & $1(5.6)$ & \\
\hline Serotoninergic antagonists & $1(9.1)$ & $4(22.2)$ & \\
\hline
\end{tabular}




\begin{tabular}{|llll|}
\hline Beta-blockers & $3(27.3)$ & $6(33.3)$ & \\
Other & 0 & $2(11.1)$ & \\
& $2(18.2)$ & $4(22.2)$ & \\
\hline Prior treatment failures, mean \pm SD & $5.1 \pm 3.1$ & $4.1 \pm 1.5$ & $\mathrm{~ns}$ \\
\hline $1-2$ & $2(10.5)$ & $2(6.5)$ & \\
$3-4$ & $9(47.4)$ & $21(63.6)$ & \\
$>4$ & $8(42.1)$ & $8(24.2)$ & \\
\hline Response to BoNT/A, $\mathrm{n}(\%)$ & $4(50.0)$ & $7(70.0)$ & $\mathrm{ns}$ \\
\hline Pts with $\geq 1$ comorbidity, $\mathrm{n}(\%)$ & $11(32.4)$ & $23(67.6)$ & $\mathrm{ns}$ \\
\hline Pts with psychiatric comorbidities, $\mathrm{n}(\%)$ & $3(15.8)$ & $7(21.2)$ & $\mathrm{ns}$ \\
\hline HIT-6 score at baseline, mean $\pm S D$ & $69.1 \pm 4.1$ & $62.7 \pm 21.5$ & $\mathrm{~ns}$ \\
\hline MIDAS score at baseline, mean $\pm S D$ & $93.7 \pm 42.0$ & $86.9 \pm 52.9$ & $\mathrm{~ns}$ \\
\hline Fremanezumab dosing regimen, $\mathrm{n}(\%)$ & & & \\
\hline Monthly & $11(57.9)$ & $33(97.1)$ & $\mathbf{0} .001$ \\
\hline Quarterly & $8(42.1)$ & $1(2.9)$ & \\
\hline
\end{tabular}

$\mathrm{CM}$ = chronic migraine; $\mathrm{BMI}$ : body mass index; MMDs: monthly migraine days; MHDs: monthly headache days; $\mathrm{NRS}=$ numerical rating scale; $\mathrm{UAs}=$ unilateral cranial autonomic symptoms; $\mathrm{MAl}=$ monthly analgesic intake; $\mathrm{MO}=$ medication overuse; $\mathrm{BoNT} / \mathrm{A}=$ onabotulinum toxin $\mathrm{A} ; \mathrm{HIT}-6$ : Headache Impact Test6; MIDAS=migraine disability assessment test.

\section{Figures}


July 28,2020

November 11, 2020

\section{7 patients eligible}

6 patients received 1 fremanezumab monthly dose

2 patients received 2 fremanezumab monthly doses 5 patients received 3 fremanezumab monthly doses 1 patient was lost to follow up

53 patients received $\geq 4$ fremanezumab monthly doses (12 weeks) and considered for effectiveness and safety analysis

\section{Figure 1}

Patients' disposition 
A

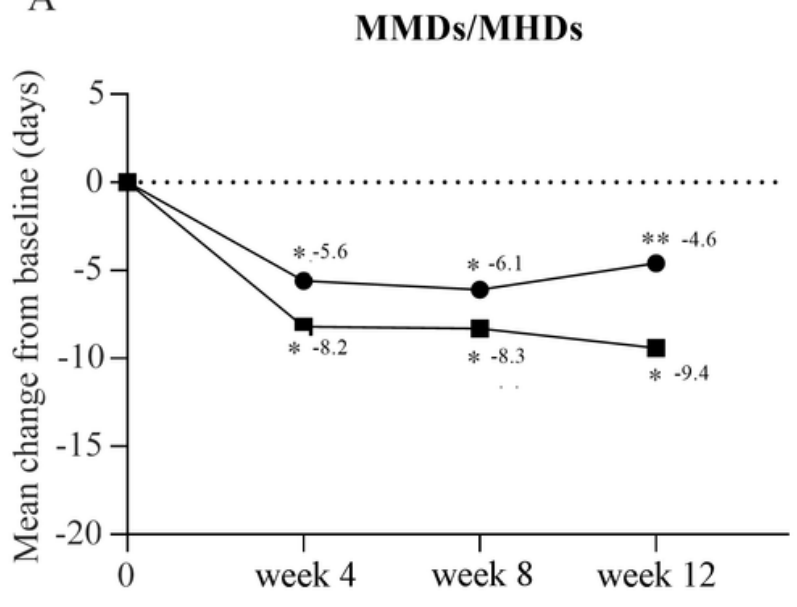

$\mathrm{C}$

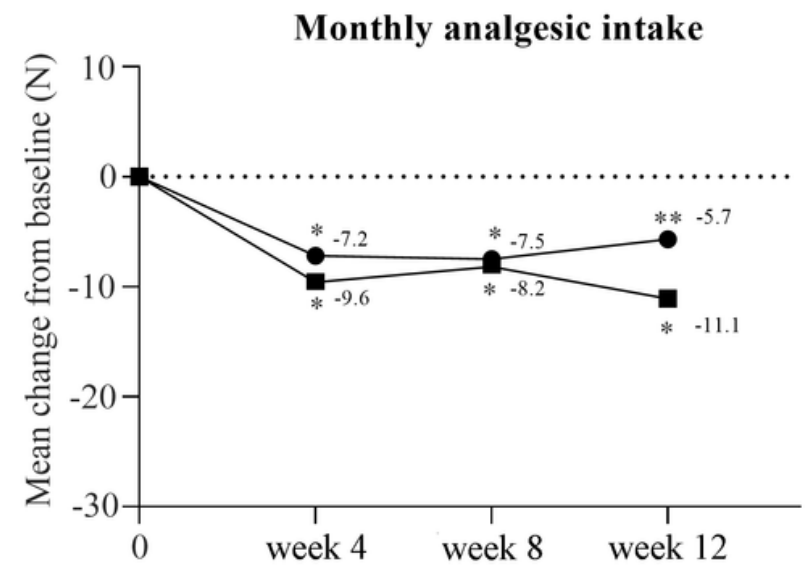

B

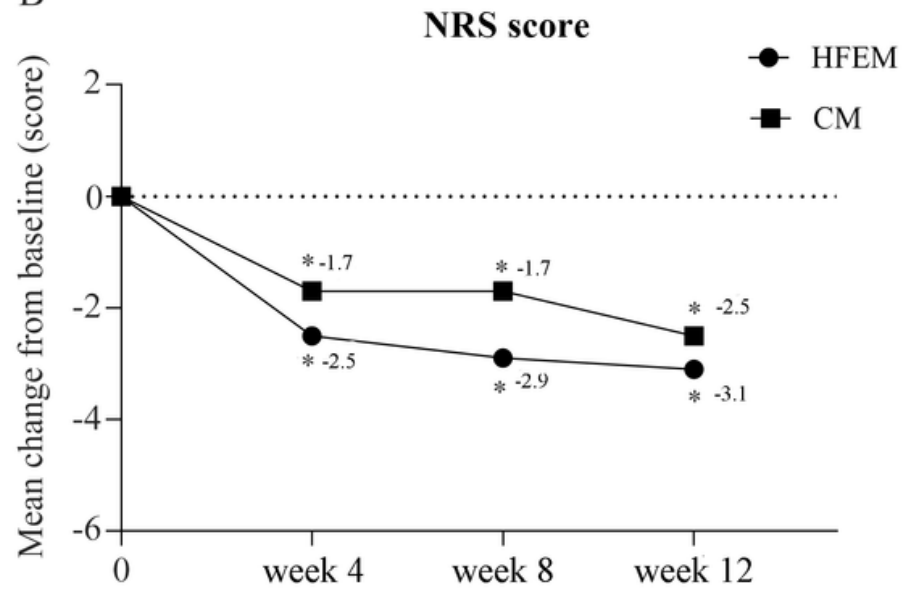

D

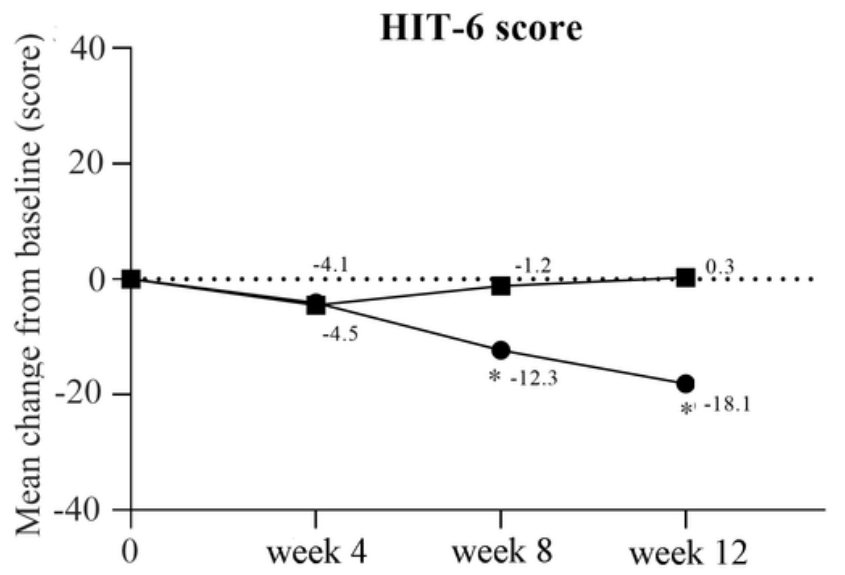

Figure 2

Mean change in (A) monthly migraine days/monthly headache days (MMDs/MHDs), (B) Numerical Rating Scale (NRS), (C) monthly analgesic intake, and (D) Headache Impact Test-6 (HIT-6) score from baseline to Week 12.

$\mathrm{CM}$, chronic migraine; HFEM, high-frequency episodic migraine 
A

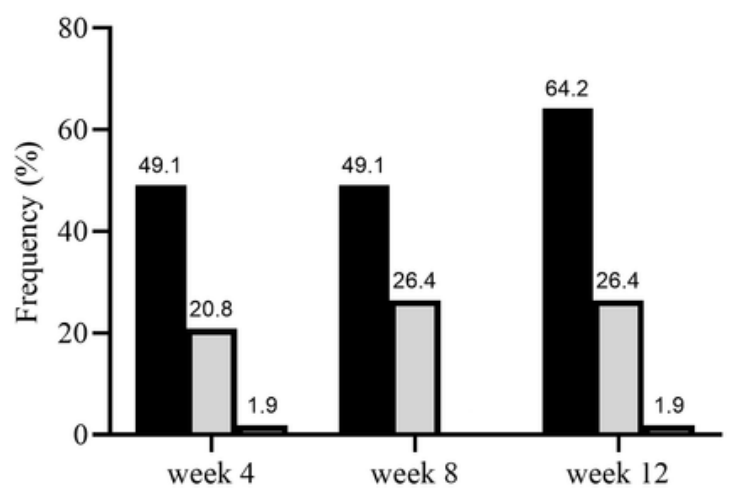

$\geqslant 50 \%$ response rate

리 response rate

100\% response rate

ALL

B
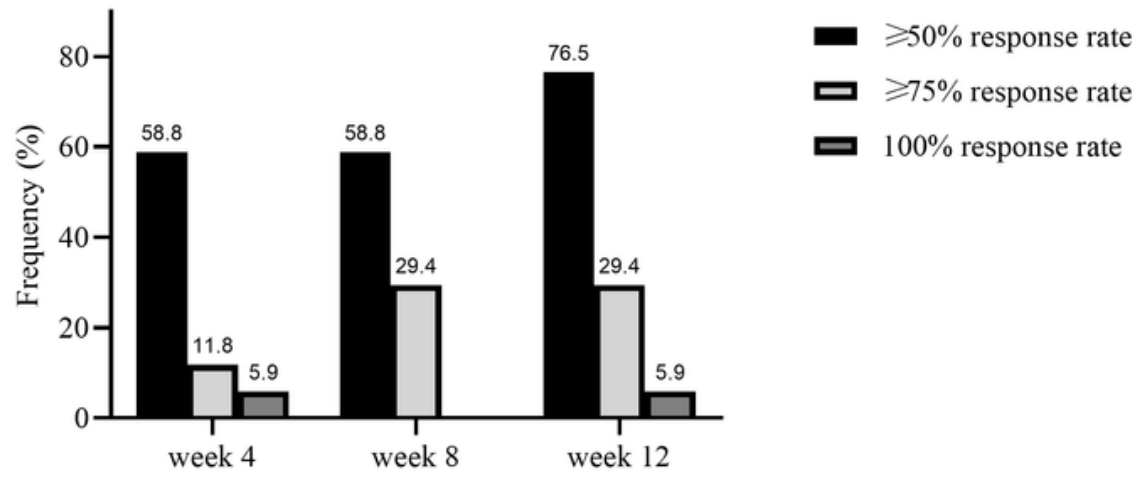

HFEM

C

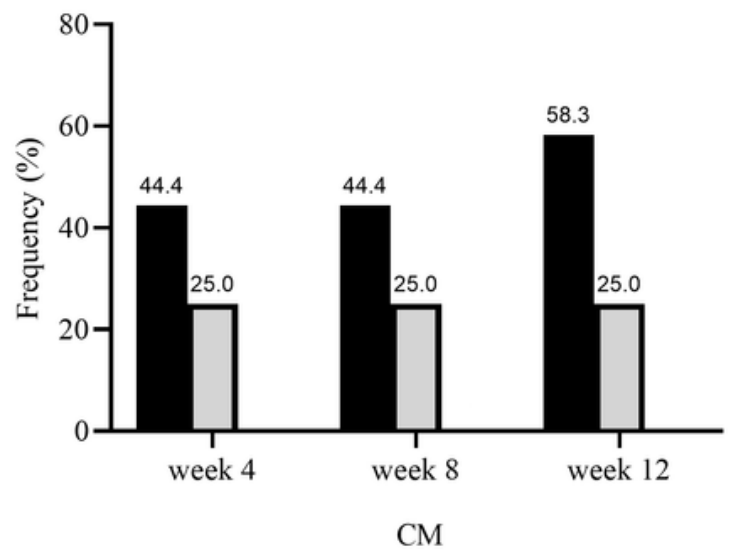

- $\geqslant 50 \%$ response rate

口 $\geqslant 75 \%$ response rate

ㅁ $100 \%$ response rate

\section{Figure 3}

Response rates at week 4, week 8, and week 12 in the global patients' population (ALL), patients with high-frequency episodic migraine (HFEM), and chronic migraine (CM).

\section{Supplementary Files}


This is a list of supplementary files associated with this preprint. Click to download.

- Supplementarytable.docx 